\title{
Radiography with Low Energy Protons Generated from Ultraintense Laser-plasma Interactions
}

\author{
Chang II Choi, Dong Hoon Lee, Byoung Hwi Kang, and Yong Kyun Kim* \\ Department of Nuclear Engineering, Hanyang University, Seoul 133-791, Korea \\ II Woo Choi, Jae Hee Sung, Chul Min Kim, I Jong Kim, Tae Jun Yu, Seong Ku Lee, \\ Ki Hong Pae, Nasr Hafz, Tae Moon Jeong, Do-Kyeong Ko, and Jongmin Lee \\ Center for Femto-Atto Science and Technology, and Advanced Photonics Research Institute, \\ GIST, Gwangju 500-712, Korea
}

(Received December 16, 2008 : revised March 11, 2009 : accepted March 11, 2009)

\begin{abstract}
In order to obtain high quality images of thin objects, we performed an experiment of proton radiography by using low energy protons generated from the interaction of an ultrashort ultraintense laser with solid targets. The protons were produced from a thin polyimide target irradiated by the laser pulse, and their maximum energy was estimated at up to $1.8 \mathrm{MeV}$. A CR-39 nuclear track detector was used as a proton radiography screen. The proton images were obtained by using an optical microscope and the spatial resolution was evaluated by a Modulation Transfer Function (MTF). We have achieved about $10 \mu \mathrm{m}$ spatial resolution of images. The obtained spatial resolution shows about $4 \sim 5$ times better value than the conventional X-ray radiography for inspection or non-destructive test (NDT) purpose.
\end{abstract}

Keywords : Proton radiography, Modulation transfer function, Ultraintense laser, CR-39, Spatial
resolution
OCIS codes : $\begin{aligned} & (140.0140) \text { Laser and laser optics; (140.3590) Lasers, titanium; (120.5630) } \\ & \text { Radiometry; (350.4990) Particles }\end{aligned}$

\section{INTRODUCTION}

The production technologies and applications of high energy protons generated by ultraintense laser are recently the hot issues in various research fields such as proton therapy, proton radiography, nuclear physics, security inspection, and so on [1]. Especially in radiography, the laser-generated protons are very useful to obtain high quality images of thin objects, because protons penetrate an object following an almost straight path and give depth distribution information of various elements in a subject. Proton radiography offers several advantages over conventional X-ray radiography for the radiography of thick, dense, dynamic systems and will provide an interesting alternative to conventional radiography. Proton radiography provides the unique possi-

*Corresponding author: ykkim4@hanyang.ac.kr bility of measuring both the density and the material composition of an object with a pulsed system [2]. Since laser-driven protons require lower cost and a smaller facility rather than accelerator-based protons, the radiography using laser-driven protons have been of interest.

There are many articles that describe the origin of the accelerated protons driven by laser but there is still a long-standing controversy on that point. Referred to the article of Okihara, et al. [3], energetic electrons of $\mathrm{MeV}$ order are generated by irradiating a thin foil target with an ultraintense laser pulse. These electrons escape from the target, and then a strong spatial electric field is generated between the fast moving electrons and the immobile ions in the target. They reported that protons are then accelerated by this strong spatial electric field. In the previous experiment, it was demonstrated that ultraintense laser-solid interactions with peak intensity of $3 \times 10^{20} \mathrm{~W} / \mathrm{cm}^{2}$ could generate energetic protons with 
maximum energy of $58 \mathrm{MeV}$ [4].

Many worldwide research teams are developing the imaging technologies using protons driven by intense laser. In previous reports, King et al. [5] obtained proton radiography images having $\sim 200 \mu \mathrm{m}$ spatial resolution by using $800 \mathrm{MeV}$ protons from an accelerator at the Los Alamos Nuclear Science Center (LANSCE). Cobble et al. [6] succeeded in producing high spatial resolution, $2 \sim 3 \mu \mathrm{m}$, proton images by using CR-39 plastic nuclear track detectors and laser-accelerated protons generated with the Los Alamos National Laboratory (LANL) Trident laser facility. Andreev et al. [7] obtained an image of a biological object, ant and grass leaf by using CR-39, radiochrome film (RCF) and protons produced by a pulsed laser. This image showed fine details such as thin hairs, of diameter about $2 \sim 3 \mu \mathrm{m}$ on the ant's body. In the report of Kar et al. [8], they obtained the images of spatial resolution of $20 \sim 30 \mu \mathrm{m}$ by using multiple layers of RCF as a proton radiography screen, and a micro-balloon object of $500 \mu \mathrm{m}$ outer diameter and $7 \mu \mathrm{m}$ wall thickness, and protons generated by the Vulcan laser system at Rutherford Appleton Laboratory (RAL).

The energetic proton generation is one of the important potential applications of high-power femtosecond laser systems. As shown in the previous studies at LANL and RAL, radiography techniques and applications using protons generated by ultraintense femtosecond lasers were actively studied. In order to apply protons generated by laser to radiography effectively, several key subjects remain such as enhancement of laser-to-proton energy conversion efficiency and optimization of proton energy [9] according to the thickness of the objects, since the proton energy determines the range in objects. More intense investigations are also needed to determine the quality of images produced by radiography. If these improvements are also achieved, the ultraintense femosecond laser facilities might replace the conventional accelerators as a source of high energy protons.

\section{EXPERIMENT AND RESULTS}

The proton radiography experiment was carried out at the Center for Femto-Atto Science and Technology (c-FAST), and Advanced Photonics Research Institute (APRI) of Gwangju Institute of Science Technology (GIST) using a $100 \mathrm{TW}$ titanium sapphire laser system [10]. Energy and duration of the laser pulse on target were $1.3 \mathrm{~J}$ and $35 \mathrm{fs}$. The protons were produced from a thin polyimide target with a thickness of $7.5,12.5$, and $25 \mu \mathrm{m}$, irradiated by the laser pulse, and were estimated at up to $1.8 \mathrm{MeV}$ of energy. The CR-39 Solid State Nuclear Track Detector (SSNTD) was used as the proton radiography screen [11]. Incident energetic charged particles left latent tracks in the CR-39, in the form of broken molecular chains and free radicals, and these latent tracks have high chemical reactivity. After chemical etching with a caustic alkali solution such as $\mathrm{NaOH}$ or $\mathrm{KOH}$, tracks forms holes [12]. The difference of holes density produces a difference of optical grey scale value. Information on the object image could be obtained by measuring the optical grey scale value of etched CR-39. The quality of proton images is then strongly dependent on spatial resolution which is mainly degraded by straggling of protons in Mylar as they pass through the object target. Using these features, we obtained proton radiography images and evaluated the quality of radiography images with the Modulation Transfer Function (MTF) method.

We manufactured a step-wedge phantom to evaluate the qualities of images and character-type phantoms to obtain on-off images. The ranges of $1.8 \mathrm{MeV}$ proton were calculated for several materials, i.e. aluminum, copper, carbon, polyethylene, etc., by using the ion range calculation program SRIM 2008 (http://www.srim.org). Mylar and polyethylene were selected as phantom material because the ranges of Mylar and polyethylene are longer than other metal materials such as aluminum and copper. As shown in FIG. 1, step-wedge phantoms were made of Mylar film with $13 \mu \mathrm{m}$ thickness and character-type phantoms were made of polyethylene with $0.5 \mathrm{~mm}$ thickness, where all protons at our experimental condition will stop inside the polyethylene.

We choose CR-39 of $1 \mathrm{~mm}$ thickness manufactured by Fukuvi Chemical Industry as a radiography screen. The CR-39 pieces with size of $5 \times 5 \mathrm{~cm}^{2}$ or $3 \times 8 \mathrm{~cm}^{2}$ were attached to the character-type phantom and the stepwedge phantom, respectively. The CR-39 pieces which

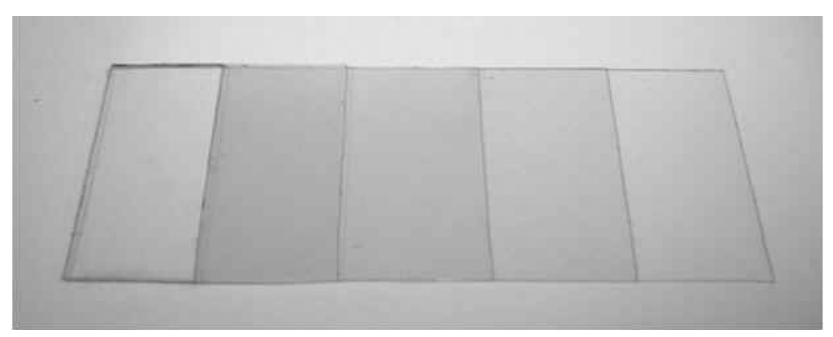

(a)

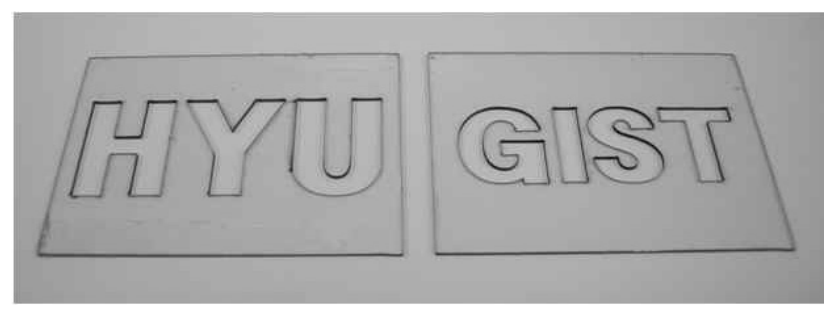

(b)

FIG. 1. Step wedge phantoms made of Mylar film with $13 \mu \mathrm{m}$ thickness (a) and character-type phantoms made of polyethylene plate with $0.5 \mathrm{~mm}$ thickness (b). 


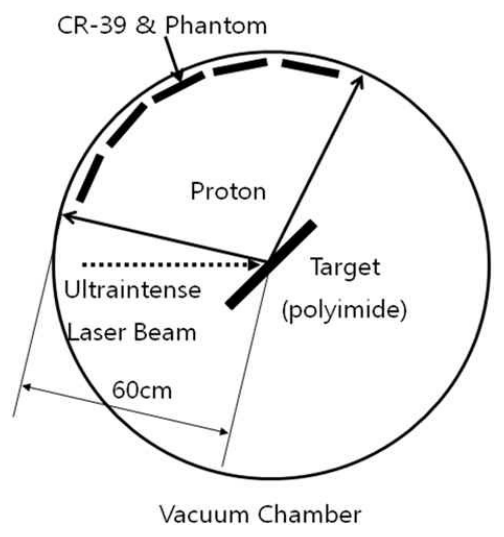

FIG. 2. Experimental setup.

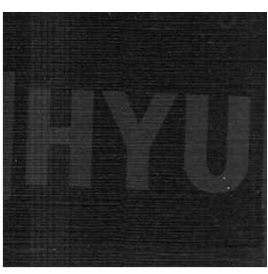

(a)

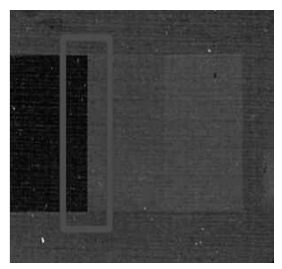

(b)

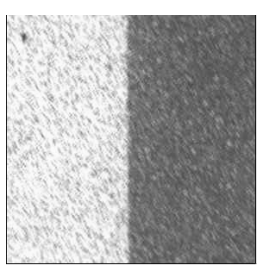

(c)
FIG. 3. The image of character phantom (a), step wedge phantom (b) after chemical etching and the obtained digital image by using an optical microscope at boundary of 39 $\mu \mathrm{m}$ and $26 \mu \mathrm{m}$ Mylar (c).

were attached to the phantoms were placed inside the experiment vacuum chamber as shown in FIG. 2.

After being exposed to protons, CR-39 pieces were etched in the $7 \mathrm{~N} \mathrm{NaOH}$ solution at $70^{\circ} \mathrm{C}$ for 2 and 4 hours $[12,13]$. In order to measure the track length of $1.8 \mathrm{MeV}$ protons, CR-39 was etched for about 16 hours in the same condition. However CR-39 of character phantom and step wedge phantom were etched for 4 hours and 2 hours, respectively, because long time etching causes track diameter expansion and the energies of protons passed through the step wedge phantom are decreased. After chemical etching, digital images were obtained by using an Olympus BX60M optical microscope and an Olympus AcquCAM II digital camera [14]. FIG. 3 shows a CR-39 piece after chemical etching and the digital image at the boundary of $39 \mu \mathrm{m}$ and $26 \mu \mathrm{m}$ Mylar phantom. The magnification of the optical microscope was $50 \mathrm{X}$ and the digital images were recorded as $1280 \times 1024$ bitmap format. The size of one pixel was about $1 \mu \mathrm{m}$.

The spatial resolution of the image obtained was evaluated by using the MTF method. Edge Spread Function (ESF) and Line Spread Function (LSF) were required to obtain MTF [15]. ESF was obtained analyzing a grey scale distribution which was acquired using XCAP image analysis software (EPIX $\mathbb{R}$ XCAP-Std V2.2.050408 for Windows, EPIX, inc. http://www.epixinc.com) at bound-

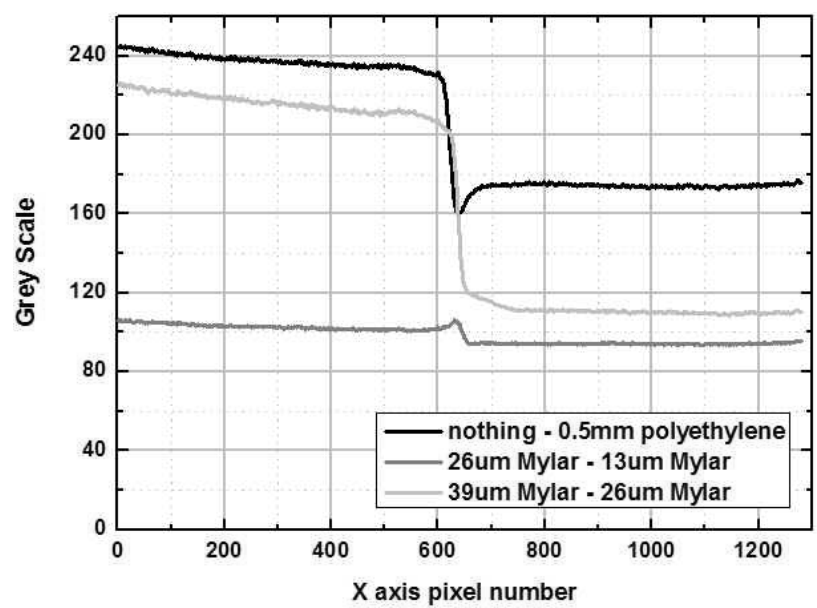

FIG. 4. Average edge spread functions of each boundary.

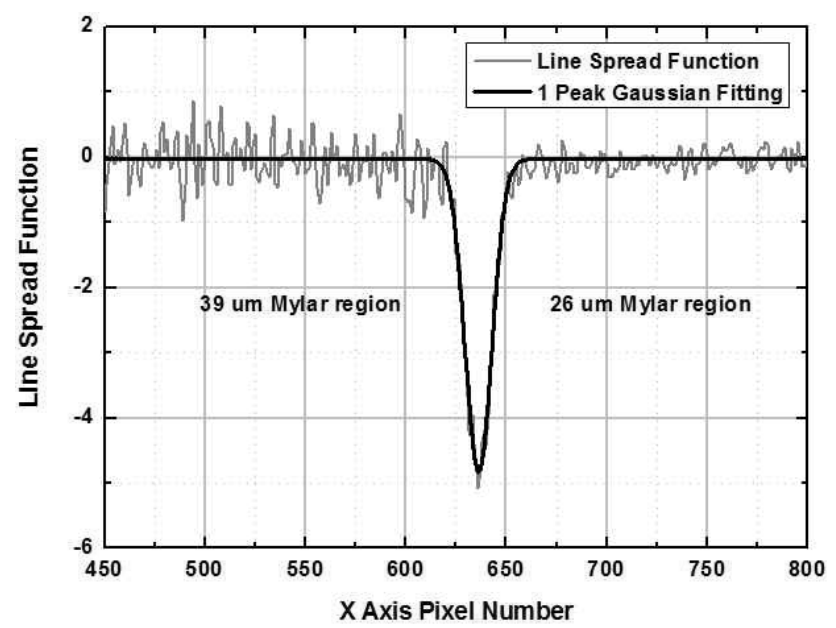

FIG. 5. Line spread function and one peak Gaussian fitting curve at boundary of $39 \mu \mathrm{m}$ and $26 \mu \mathrm{m}$ Mylar.

aries between no material and $0.5 \mathrm{~mm}$ polyethylene, between $26 \mu \mathrm{m}$ and $13 \mu \mathrm{m}$ Mylar, between $39 \mu \mathrm{m}$ and $26 \mu \mathrm{m}$ Mylar. FIG. 4 shows ESF at each boundary. The grey scale difference at the boundary of $39 \mu \mathrm{m}$ and 26 $\mu \mathrm{m}$ Mylar was larger than that between $26 \mu \mathrm{m}$ and 13 $\mu \mathrm{m}$ Mylar, because the number of protons passing through the $39 \mu \mathrm{m}$ Mylar was much smaller than that of $26 \mu \mathrm{m}$ Mylar compared with that of $26 \mu \mathrm{m}$ and $13 \mu \mathrm{m}$ Mylar.

The LSF is a function of ESF differentiated as

$$
\operatorname{LSF}(n)=\frac{d}{d x} \operatorname{ESF}(n)=\frac{\operatorname{ESF}(n+1)-\operatorname{ESF}(n-1)}{2}
$$

where $\operatorname{LSF}(n), \operatorname{ESF}(n)$ are the value of the nth pixel in digital image. FIG. 5 shows an LSF, which was fitted as one Gaussian distribution to acquire MTF curves.

The MTF was acquired by analyzing the LSF through the fast Fourier transform method and the curves were normalized with the following equation as 


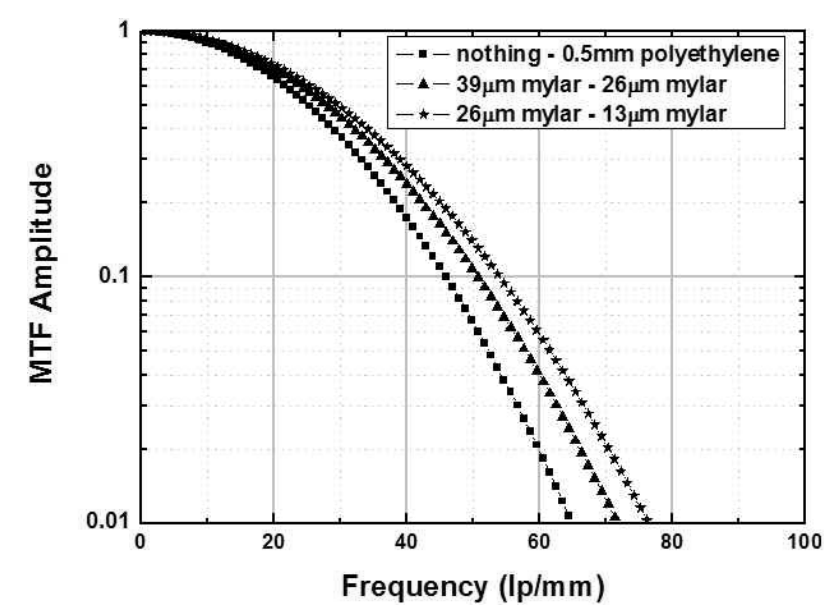

FIG. 6. Modulation transfer functions of each boundary.

TABLE 1. Spatial resolutions at each boundary.

\begin{tabular}{c|c|c}
\hline \hline Region & $\begin{array}{c}\text { Resolution } \\
(\mathrm{lp} / \mathrm{mm})\end{array}$ & $\begin{array}{c}\text { Resolution } \\
(\mu \mathrm{m})\end{array}$ \\
\hline $\begin{array}{c}\text { no material }-0.5 \mathrm{~mm} \\
\text { polyethylene }\end{array}$ & 45.9 & 10.9 \\
\hline $\begin{array}{c}26 \mu \mathrm{m} \text { Mylar }-13 \mu \mathrm{m} \\
\text { Mylar }\end{array}$ & 50.7 & 9.9 \\
\hline $39 \mu \mathrm{m}$ Mylar $-26 \mu \mathrm{m}$ \\
Mylar
\end{tabular}

※ lp : line pair

$$
\operatorname{MTF}(u)=\frac{|L(u)|}{L(0)}
$$

where $\mathrm{L}(\mathrm{u})$ is value of the LSF which is Fourier transformed. FIG. 6 shows MTF curves at each boundary.

We analyzed the MTF at the Rayleigh limit, where the MTF amplitude value is 0.1 , and the spatial resolutions were $45.9,50.7$ and $54.0 \mathrm{lp} / \mathrm{mm}$ at each boundary, which correspond to $10.9,9.9$, and $9.3 \mu \mathrm{m}$, respectively. TABLE 1 shows the spatial resolution values at each boundary.

\section{DISCUSSION AND CONCLUSION}

We have studied radiography using low energy protons which were generated from a solid target irradiated by an ultrashort ultraintense titanium sapphire laser. The CR-39 nuclear track detector was used for proton imaging. The proton images were obtained by quantitative analysis of grey scale of CR-39 detector. The resulting spatial resolution was evaluated as $\sim 10 \mu \mathrm{m}$ and is $4 \sim 5$ times better than conventional X-ray radiography images of 35 50 $\mathrm{mm}$, i.e. $10 \sim 15 \mathrm{lp} / \mathrm{mm}$ for inspection or NDT purpose. Compared to the experiment at Los Alamos
National Laboratory Trident laser facility [6], our results are about 3 times poorer. It can be considered to be due to the differences in the chemical etching condition of CR-39, the evaluation method, and the target material. The results of Andreev et al. [7] showed more precise image than our result. It can be considered due to the differences in the proton detector and the evaluation method. Even in the present simple experiment, we could obtain a considerable proton radiography image of $\sim 10$ $\mu \mathrm{m}$ spatial resolution. It is expected to improve the quality of images by optimizing the imaging procedure.

We used a method to analyze the qualities of proton radiography images quantitatively using MTF and demonstrated the images' experimental possibility of obtaining proton radiography from ultraintense femtosecond laser facility at c-FAST and APRI of GIST, Korea. In order to improve the quality of images and calibrate the energy of protons, further experiments by using the radiochrome film and the accelerator driven protons with known energy will be necessary. When higher energy protons are available, more enhanced proton radiography images can be obtained reasonably.

\section{ACKNOWLEDGMENT}

This work was supported by the Ministry of Knowledge and Economy of Korea through the Ultrashort Quantum Beam Facility Program, and partially supported by the Basic Atomic Energy Research Institute (BAERI), nuclear R\&D program of MEST, Korea.

\section{REFERENCES}

1. D. Umstadter, "Relativistic laser-plasma interactions," J. Phys. D: Appl. Phys. 36, R151-R165 (2003).

2. J. A. Cookson, "Radiography with protons," Naturwissenschaften 61, 184-191 (1974).

3. S. Okihara, Y. Sentoku, K. Sueda, S. Shimzu, F. Sato, et al., "Energetic protons generation in a thin plastic foil irradiated by intense femtosecond lasers," J. Nucl. Sci. Technol. 39, 1-5 (2002), and references in therein.

4. R. A. Snavely, M. H. Key, S. P. Hatchett, T. E. Cowan, M. Roth, et al., "Intense high-energy proton beams from petawatt-laser irradiation of solids," Phys. Rev. Lett. 85, 2945-2948 (2000).

5. N. S. P. King, E. Ables, K. Adams, K. R. Alrick, J. F. Amann, et al., "An 800-MeV proton radiography facility for dynamic experiments," Nucl. Instrum. Methods Phys. Res. A 424, 84-91 (1999).

6. J. A. Cobble, R. P. Johnson, T. E. Cowan, N. R.-Le Galloudec, and M. Allen, "High resolution laser-driven proton radiography,” J. Appl. Phys. 92, 1775-1779 (2002).

7. A. A. Andreev, V. A. Komarov, K. Yu. Platonov, and A. V. Charukhchev, "Spatial resolving power of laser plasma ionography,” Tech. Phys. Lett. 33, 239-243 (2007). 
8. S. Kar, M. Borghesi, P. Audebert, A. Benuzzi-Mounaix, T. Boehly, et al., "Modeling of laser-driven proton radiography of dense matter," High Energy Density Phys. 4, 26-40 (2008).

9. I. W. Choi, C. M. Kim, T. M. Jeong, T. J. Yu, J. H. Sung, et al., "Proton generation with 3-\% energy conversion efficiency," in Proc. OSK Summer Meeting (Phoenixpark, Korea, Jul. 2008), pp. 155-156.

10. J. H. Sung, T. J. Yu, S. K. Lee, T. M. Jeong, I. W. Choi, et al., "Development of the ultrashort high-power femtosecond lasers at APRI," in Proc. 4th ASILS 2008 (GIST, Korea, Nov. 2008), pp. 68.

11. B. A. De Souza, S. C. Cabral, and R. T. Lopes, "Alpha particle radiography with the CR-39 nuclear track detector," Radiat. Meas. 24, 187-192 (1994).

12. S. Kar, M. Borghesi, L. Romagnani, S. Takahashi, A. Zayats, et al., "Analysis of latent tracks for $\mathrm{MeV}$ protons in CR-39,” J. Appl. Phys. 101, 044510 (2007).

13. M. A. Rana and I. E. Qureshi, "Studies of CR-39 etch rates," Nucl. Instrum. Methods Phys. Res. B 198, 129-134 (2002).

14. Y. L. Law, D. Nikezic, and K. N. Yu, "Optical appearance of alpha-particle tracks in CR-39 SSNTDs," Radiat. Meas. 43, S128-S131 (2008).

15. Anthony Brinton Wolbarst, Physics of Radiology (Prentice Hall International, London, UK, 1993) Chapters 18 and 24. 